\title{
Initial Reliability and Validity Testing of the DESTINY Stroke and Large Vessel Screening Tool
}

\author{
https://doi.org/10.37719/jhcs.2020.v2i2.oa004
}

DANIEL NYANCHO, MPH

https://orcid.org/0000-0002-7753-5244

RYAN CHEUNG, DO

https://orcid.org/0000-0003-0292-5050

AARDHRA M. VENKATACHALAM, MPH

https://orcid.org/0000-0001-8449-042X

FOLEFAC D. ATEM, PhD

https://orcid.org/0000-0002-1142-7806
SONJA E. STUTZMAN, PhD

https://orcid.org/0000-0002-3121-2829

DAIWAI M. OLSON, PhD, RN, FNCS

https://orcid.org/0000-0002-9280-078X

DEBORAH DIERCKS, MD

https://orcid.org/0000-0003-2043-7405

MARK JOHNSON, MD

https://orcid.org/0000-0002-1625-6997

ROBERTA NOVAKOVIC-WHITE, MD

https://orcid.org/0000-0003-3220-3323

University of Texas Southwestern Medical Center, Dallas, Texas, USA

Corresponding author's email: DaiWai.OIson@UTSouthwestern.edu

\section{Abstract}

Background: Screening is a valuable tool in emergency medicine for triaging, activation of protocols, and resource allocation. The Dallas Emergency Department Screening Tool to Identify Stroke (DESTINY) was developed to screen for all stroke subtypes, with improved sensitivity for posterior circulation stroke and large vessel occlusion (LVO). The main purpose of this study was to evaluate how reliably the tool could be taught and used by ED Nurses.

Methods: This is Phase 1 of a multi-phase study to evaluate the DESTINY tool. A vascular neurologist retrospectively reviewed 409 stroke code activations between January and December of 2018. Thirty descriptive vignettes were composed: right anterior circulation stroke $(n=5)$, left anterior circulation stroke $(n=5)$, posterior circulation stroke $(n=5)$, TIA and stroke mimic $(n=6)$, non-LVO stroke $(n=5)$, and hemorrhagic stroke $(n=4)$. Fifteen emergency medicine registered nurses used the DESTINY tool to screen the vignettes following the stroke education and DESTINY training module. 
Results: Individual Pearson Correlation Coefficients (PCC) were calculated for each nurse compared to the vascular neurologist (range: 0.43860 to 0.96966 ; mean: $0.8746 \pm 0.1484$ ). All individual PCC were statistically significant except for one [PCC of $0.43860(p=0.0153)]$. Cronbach's Alpha scores were very similar across multiple raters (standardized alpha for nurse raters: 0.9853 ). Omnibus test to compare DESTINY scores by stroke type demonstrated left anterior LVO and mimic/TIA stroke had few outlier observations, however, poorly differentiated hemorrhagic from ischemic stroke.

Conclusion: As demonstrated by high interrater reliability, the DESTINY tool can be effectively taught to ED nurses using clinical vignettes. The DESTINY tool is good at differentiating non-LVO, anterior LVO, mimics, and TIAs; while the ability to distinguish between anterior LVO/posterior LVO and ischemic/hemorrhagic stroke was sub-optimal. Utilizing the lessons learned in the early version of the DESTINY screening tool we expect that the high-reliability scores will continue to improve in future prospective studies.

Keywords: stroke, large vessel occlusion, screening tool, validity, reliability

\section{Introduction}

- The incidence of acute ischemic stroke (AIS) remains high and is among the leading causes of disability and mortality in the United States and worldwide (Benjamin et al., 2019; Krishnamurthi et al., 2013). The presence of a large vessel occlusion (LVO) has been associated with significantly worse outcomes (Zhu et al., 2014), increasing the odds of mortality by 4.5-fold within 6 months (Smith et al., 2013). Meanwhile, the absence of LVO increases the odds of a good outcome measured by the modified Rankin Scale (mRS) within 6-months (Smith et al., 2013). The most recent guidelines clarify that thrombolytic therapy (e.g. Alteplase or Tenecteplase) be given even when endovascular thrombectomy is planned (Powers et al., 2019). Due to the suboptimal rates of recanalization of a LVO following the administration of intravenous (IV) alteplase and the superiority of thrombectomy combined with best medical management, guidelines have adopted EVT as the standard treatment in eligible patients with LVO up to 24 hours from the last known well (LKW) (Powers et al., 2019; Lawrence et al., 2018). Hence, early identification of LVO is critical for proper treatment selection and triage (Hill et al., 2020).

Despite some terminology variation, the most common definitions of LVO include the internal carotid artery (ICA), proximal middle cerebral artery (M1), distal middle cerebral artery territory (M2), and basilar artery (BA). Additional arterial territory with variable acceptance as LVO includes the vertebral artery (VA), posterior cerebral artery (PCA), and anterior cerebral artery (ACA). Screening for LVO currently includes neurological assessment, CT angiography, and MR angiography (Ver Hage et al., 2018; Beume et al., 2018; Lakomkin et al., 2019; Rennert et al., 2019). Depending on the classification used, angiographic studies have shown that LVO accounts for $31-46 \%$ of ischemic strokes and 13\% of transient ischemic attacks (TIA) (Smith et al., 2009; Beumer et al., 2016). 
EVT treatment benefits diminish as the time between stroke onset and treatment increases. Ergo it is important that stroke systems of care rapidly identify patients with a LVO in the prehospital setting for proper triage of these patients to the highest level of care. Time is lost when a patient with a LVO is transported to a lower-level stroke facility that lacks the resources to provide the necessary treatment (Teleb et al., 2017). Currently, emergency medical services (EMS) first responders perform one screening tool to identify stroke and then apply a second stroke severity tool to identify those patients most likely to have a LVO. Although there are numerous assessment tools used to predict the presence of LVO in stroke patients, a systematic review showed that some instruments did not predict LVO with both high sensitivity and high specificity (Smith et al., 2018). Additionally, many of the stroke severity tools have low sensitivity for posterior circulation strokes. As such, more prospective studies were recommended to assess the accuracy of LVO prediction instruments (Powers et al., 2019; Smith et al., 2018)

The National Institutes of Health Stroke Scale (NIHSS) is widely used as a clinical assessment tool to evaluate the severity of stroke patients, predict patient outcomes, and determine appropriateness for treatment. The NIHSS objectively quantifies stroke impairment on a 0 to a 42point scale and ranks the severity of the stroke from minor to moderate to severe. Patients with a LVO have a higher NIHSS score compared to those without and are associated with a 7.8-point increase (Smith et al., 2009). However, NIHSS is only a low predictor of LVO, as the tool was not intended to screen for LVO but to quantify the severity of the stroke. The precision of NIHSS in screening for LVO is low, especially when the NIHSS is less than 20 (Beumer et al., 2016). Additionally, the NIHSS takes regular training to perform accurately and is more time consuming than the rapid assessment tools used in the field.

Much like in the prehospital setting, relying on the NIHSS as a rapid assessment can lose valuable time and sensitivity in the Emergency Department (ED) when attempting to quickly identify stroke, mobilize the appropriate teams, and outline the imaging necessary for work-up. A patient suspected of having an AIS with or without LVO in the early and late window necessitates different imaging modalities. Thus, there is the potential for increased radiation and contrast exposure when these studies are not performed discriminately. As the expectation for performance metrics increases, it becomes harder to hit the target times without having imaging that is performed within minutes from the patient arriving at the ED. Thus, a rapid triage assessment tool that can identify the type of stroke, predict LVO, and has appropriate sensitivity for posterior circulation strokes could save time in the ED and facilitate faster treatment times.

\section{Methods}

The Dallas Emergency Department Screening Tool to Identify Stroke (DESTINY) was developed as a rapid assessment tool to screen for all strokes while having improved sensitivity for 
posterior circulation stroke as well as identify those caused by LVO. Approval for this phase 1 study was obtained from the Local University Institutional Review Board. Prior to developing the DESTINY screening tool, commonalities, and differences among stroke assessment tools were reviewed by physicians and nurses in vascular neurology, interventional radiology, neurosurgery, and emergency medicine. From these instruments (Table 1) key items were retained, new items were added, and then organized for flow. Mock-up instruments were field-tested by asking ED nurses to complete the tool as if they were assessing a patient (e.g., imagine your last stroke patient and complete this tool). The final DESTINY instrument was then made available in print form.

Table 1. Previous stroke assessment tools used, and new elements of DESTINY

\begin{tabular}{|c|c|}
\hline Scales & What was used for DESTINY \\
\hline NIHSS & Used as control \\
\hline VAN12 & Facial palsy, limb weakness, neglect \\
\hline CPHSS 17 & Difficulty controlling a limb, speech disturbance; arm weakness \\
\hline ROSIER ${ }^{18}$ & Seizure activity, speech disturbance, vision loss \\
\hline CSTAT $^{19}$ & $\begin{array}{l}\text { Arm weakness, gaze deviation, level of consciousness/following } \\
\text { commands, hemi sensory loss }\end{array}$ \\
\hline New in DESTINY & $\begin{array}{l}\text { Pain as chief complaint, Did symptoms resolve, symptoms start with } \\
\text { headache sudden onset, loss of balance chief complaint }\end{array}$ \\
\hline
\end{tabular}

NIHSS $=$ National Institute of Health Stroke Scale; VAN=Vision Aphasia Neglect; CPHSS=Cincinnati prehospital stroke scale; ROSIER=Recognition of Stroke in the Emergency Room; CSTAT=Cincinnati Stroke Triage Assessment Tool

Case study subjects were developed by a clinical team composed of experts from emergency medicine, vascular neurology, and neurointerventional radiology. Clinicians retrospectively reviewed 409 cases (stroke code activations) between January and December of 2018. From these cases, 30 were selected to represent common diagnoses: right anterior circulation stroke $(n=5)$, left anterior circulation stroke $(n=5)$, posterior circulation stroke $(n=5)$, TIA and stroke mimic $(n=6)$, non-LVO stroke $(n=5)$, and hemorrhagic stroke $(n=4)$. Cases were then written as descriptive vignettes (Figure 1). When information necessary for the DESTINY screening tool was unavailable in the health record best clinical judgment was used to create the summary. The clinical vignettes were de-identified and only the physician investigator and study team creating the vignettes knew the health protected information.

\section{Example of a clinical vignette}

"The patient is a 68-year-old woman with a past medical history of hypertension and allergies who presents to the ED on 06/25/2017 at 1642 with sudden onset of $L$ sided weakness while flying from Atlanta to Las Vegas for a conference that started at 1531. The plane made an emergency landing at DFW and she was taken to the community-based hospital. 
On exam, her speech is clear. She can state her age and the month correctly. She can name objects appropriately and can follow commands by closing her eyes and open and closing her hand. Gaze is midline. Visual fields are full in 4 quadrants. She has a mild droop to the left side of her face. She drifts the left arm when attempting to hold it up for 10 seconds. She does not feel any touch to the left side of her face, arm, and leg. When both sides of her vision are tested simultaneously she only acknowledges the right side. Finger to the nose is normal."

In total, 15 emergency medicine registered nurses working in an urban area, with experience in treating strokes at a large academic hospital (Clements University Hospital [CUH]) and a community-based hospital (Parkland Medical Hospital [PMH]), volunteered to participate in the study. The volunteers were provided with an education module that focused on stroke, neurological exam and training on the DESTINY screening tool. Following the training, the volunteers were provided the 30 clinical vignettes arranged in random order, de-identified to health protected information as well as the subcategory of stroke etiology. The nurses were instructed to independently review the clinical vignettes and score each case with the DESTINY screening tool. The nurses had one week to return their results. A comment section was provided for the nurses to evaluate the DESTINY screening tool and discuss any areas they found ambiguous or confusing.

The 30 clinical vignettes were scored using the DESTINY tool by 15 emergency department nurses and a physician investigator (MD-PI) stroke specialist. Statistical analysis of the data was done on SAS v9.4 on Microsoft Windows. The scoring produced 16x30 data points that were entered into an electronic spreadsheet. A simple regression analysis was used to attain the Pearson correlation coefficient, which describes the association of the various vignettes scores in predicting the subcategory or stroke etiology.

\section{Results}

15 nurses participated in two separate emergency departments. Each nurse scored 30 clinical vignettes such that 450 scored evaluations were included in the analysis. Individual Pearson Correlation Coefficients (PCC) was calculated for each nurse in comparison to the medical provider (MD-PI reference) and ranged from 0.43860 to 0.96966 . All individual PCC were statistically significant $(\alpha<0.005)$ except for a community-based nurse with a PCC of $0.43860(p=0.0153)$. The average PCC value is $0.8746 \pm 0.1484$.

Cronbach alpha scores (a) measures the degree to which DESTINY scores were reliably comparable to all raters. ${ }^{15}$ Determination of the Cronbach's alpha score (CAS) used multiple administrations of the same test for different vignettes by different raters. Therefore, this method uses CAS to determine the reliability of DESTINY between various raters, in other words, the interrater reliability. Models were initially constructed to evaluate Cronbach's Alpha Scores with the physician $\mathrm{PI}$ as the reference. The standardized alpha for the nurse raters from the academic hospital and the community-based hospital was 0.9853 
Table 2. Pearson's correlations and Cronbach's Alpha Score for Academic and Community Based Urban Hospitals

\begin{tabular}{lcc}
\hline Hospital Rater & Pearson Correlation Coefficient & p-value \\
\hline Nurse 1 & 0.92549 & $<0.0001$ \\
Nurse 2 & 0.96966 & $<0.0001$ \\
Nurse 3 & 0.86412 & $<0.0001$ \\
Nurse 4 & 0.94248 & $<0.0001$ \\
Nurse 5 & 0.60990 & 0.0003 \\
Nurse 6 & 0.94437 & $<0.0001$ \\
Nurse 7 & 0.93211 & $<0.0001$ \\
Nurse 8 & 0.93575 & $<0.0001$ \\
Nurse 9 & 0.87591 & $<0.0001$ \\
Nurse 10 & 0.94231 & $<0.0001$ \\
Nurse 11 & 0.95519 & $<0.0001$ \\
Nurse 12 & 0.91917 & $<0.0001$ \\
Nurse 13 & 0.92006 & $<0.0001$ \\
Nurse 14 & 0.43860 & 0.0153 \\
Nurse 15 & 0.94379 & $<0.0001$ \\
\hline \multicolumn{2}{|}{} \\
\hline Pearson correlation Coefficient Mean & $0.8746 \pm 0.1484$ \\
PCC Range & & $0.43860-0.96966$ \\
Cronbach's Coefficient Alpha & 0.985282
\end{tabular}

A total of 480 observations were used for an omnibus test to compare DESTINY scores by stroke type because each vignette represents a stroke etiology; left anterior LVO $n=80$ mean= $5.400 \pm 1.548$, right anterior LVO $n=80$ mean $=5.563 \pm 1.483$, posterior LVO $n=80$ mean= $5.400 \pm 3.366$, hemorrhagic $n=64$ mean $=4.922 \pm 2.651$, non-LVO $n=80$ mean= $2.889 \pm 1.49$, and $T I A$ $\mathrm{n}=64$ mean $=1.667 \pm 1.463$. Left anterior LVO and mimic/TIA stroke had a few outlier observations

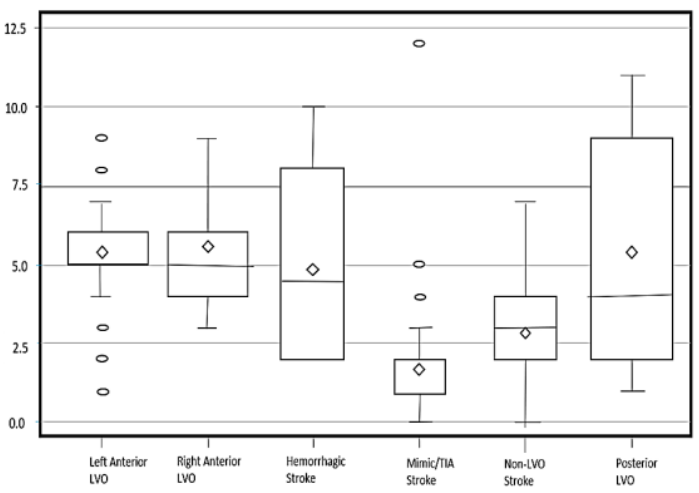

Figure 2. Distribution of scores by diagnostic category LVO=Large vessel occlusion; TIA=transient ischemic attack 


\section{Discussion}

Screening tools are valued in the clinical setting for their ability to detect signs of a disease in an individual to reduce the chances for mortality or morbidity. Screening is also a valuable tool in emergency medicine for the triaging, activation of protocols, and resource allocation. Aside from having high sensitivity and specificity for stroke and LVO, an effective screening tool must be one that is simple to perform and teach to providers of various medical backgrounds. The results must then be reliable among clinicians (including EMS, triage nurses, and ED physicians) in a variety of settings. This ensures that the patients are properly triaged promptly to the appropriate care.

The validity of a new tool is dependent on the reliability of the tool (Buelow et al., 2016). Cronbach's alpha score is a widely used objective measure of reliability test to determine the degree of the interrelatedness of test items or raters in an assessment or tool (Tavakol \& Dennick, 2011). CAS analysis showed that DESTINY has high internal consistency $(\alpha=0.985)$ indicating that the scores are very similar across multiple raters. Therefore, the DESTINY tool has high interrater reliability when applied to potential stroke patients with a variety of presentations.

As with any new instrument, there is room for improvement. Future modifications will aim to reduce limitations associated with definitions and terminology that were more open to interpretation than we had recognized. These modifications may further enhance internal consistency. Limitations of generalizability are currently being examined as the instrument is now the standard of care at another institution. These data will be evaluated in a future manuscript.

In this retrospective phase 1 study, the tool was shown to be effectively taught to ED nurses who had familiarity with stroke assessment before using DESTINY. The tool was applied to our patient population consistently and reliably. Individual PCCs were all positive and showed a moderate to strong correlation to the MD-PI reference. This indicates how effective the current DESTINY educational teaching module is for raters of different backgrounds. All individual PCC were statistically significant $(\alpha<0.005)$ except for a single PMH-ED nurse $(P C C=0.43860, p=0.0153)$.

The omnibus test provided the average score ranges between all raters for each stroke etiology. A screening tool with a clear benchmark for each possible stroke type is essential for an improved and rapid stroke care system to achieve appropriate stroke identification, triage, and treatment (Middleton et al., 2015). In this study, the DESTINY tool is good at differentiating non-LVO strokes as well as cases that were not strokes such as mimics and TIAs; DESTINY had good construct validity for non-strokes. Although DESTINY's ability to distinguish between anterior LVO and posterior LVO was sub-optimal, the upper and lower boundaries for anterior LVO is quite adequate for differentiation. However, the DESTINY screening tool poorly differentiated hemorrhagic from ischemic stroke. 
The nurses had the opportunity to critique the questions. Based on their evaluations, future modifications to DESTINY address an ambiguous question related to posterior LVO stroke, which was also associated with a higher rate of error in their responses compared to MD-PI reference. Future modifications to DESTINY address the ambiguous language in the screening tool and addressed test items to better differentiate anterior LVO from posterior LVO and ischemic from hemorrhagic stroke. Although, the main intent of the screening tool is to identify ischemic stroke and its subtypes rather than screen for hemorrhagic stroke.

\section{Limitations}

One recognized limitation of this study includes only testing the interrater reliability of the DESTINY tool and not comparing it to other published stroke and LVO screening tools. Comparing DESTINY to other screening tools performed by the same examiner would yield a more effective comparison as to the efficacy of DESTINY. This is planned for a future study. Also, our sample included nurses who were already familiar with stroke assessment and future studies should address clinicians with varying degrees of familiarity assessing stroke.

An additional significant limitation of this study is the application of the DESTINY tool to written selected cases for this study. DESTINY was created for the triage of potential stroke patients presenting to an ED in a time-critical period and assessing for high reliability in a live setting would be most fitting. This is the first among several planned prospective studies to evaluate DESTINY in a real-world environment to see if these findings remain robust.

A Cronbach alpha score of 0.9853 is extremely high. It is worth mentioning that Cronbach's alpha is neither a test of dimensionality (unidimensionality or multidimensionality) nor a measure of validity without capturing any confounder. However, it is a strong measure of internal consistency or validity. So a high alpha simply implies high consistency. 15 According to the omnibus test analysis, this version of DESTINY can accurately distinguish between stroke mimics/TIA and LVO but shows poor resolution between ischemic and hemorrhagic strokes. This may indicate that DESTINY is measuring LVO in addition to ischemic and hemorrhagic strokes (i.e. multidimensional). Future studies will address this issue and update DESTINY as necessary.

\section{Conclusion}

Given the broad nature of stroke patients and their myriad presentations, an ideal stroke screening tool must have high sensitivity and specificity for strokes of all subtypes. DESTINY is a multi-phase study and this study concludes phase 1 . The main purpose of this study was to evaluate the usability of the tool and how reliably it could be taught and deployed by ED nurses. The results show that the scores among examiners could be reliable following an educational training module. In 
this study, the tool was only retrospectively applied to de-identified clinical vignettes from thirty patients representing the varied presentations of stroke. Utilizing the lessons learned and deficiencies in the early version of the DESTINY screening tool, we expect that the high-reliability scores will continue to improve. Future prospective studies will further evaluate for weaknesses inherent in the DESTINY screening tool for identifying stroke and differentiating between the stroke subtypes, as well as the feasibility of applying the prospective tool in a real emergency setting.

\section{Conflict of Interest}

The authors have no conflict of interest to disclose.

\section{Funding}

The study received funding from the UT Southwestern Medical Center.

\section{Author Contribution}

Daniel Nyancho: conceptualization of the study, acquisition of data, analysis of data, drafting of manuscript, critical revisions. Ryan Cheung: data acquisition, drafting of manuscript, critical revisions. Aardhra M. Venkatachalam: analysis of data, critical revisions. Folefac D. Atem: analysis of data, critical revisions. Sonja E. Stutzman: conceptualization of the study, data acquisition, drafting of manuscript, critical revisions. DaiWai M. Olson: drafting of manuscript, design of the study, interpretation of data, critical revisions. Deborah Diercks: interpretation of data, critical revisions. Mark Johnson: interpretation of data, critical revisions. Roberta Novakovic-White: conceptualization of the study, study design, data acquisition, interpretation of data, drafting of manuscript, critical revisions.

\section{References}

Benjamin, E.J., Muntner, P., Alonso, A., Bittencourt, C., Callway, C., Carson, A., Chamberlain, A., Chang, A., Cheng, S., Das, S., Delling, F., Djousse, L., Elkind, M., Ferguson, J., Fornage, M., Jordan, L., Khan, S., Kissela, B., Knutson, K., Kwan, T., \& Virani, S. (2019). Heart Disease and Stroke Statistics; 2019 Update: A Report From the American Heart Association. Circulation, 139(10):e56-e528. https://doi.org/10.1161/CIR.0000000000000659

Beumer, D., Mulder, M, J. H. L., Saiedie, G., Fonville, S., van Oostenbrugge, van Zwam, W., Homburg, P., van der Lugt, A., Dippel, D. (2016). The occurrence of intracranial large vessel occlusion in consecutive, non-referred patients with acute ischemic stroke. Neurovascular Imaging, 2(1):11. https://doi.org/10.1186/s40809-016-0022-5 
Beume L-A., Hieber, M., Kaller, C. P., Nitschke, K., Bardutzky, J., Urbach, H., Weiller, C., Rijntjes, M. (2018).. Large Vessel Occlusion in Acute Stroke. Stroke, 49(10):2323-2329. https://doi.org/10.1161/STROKEAHA.118.022253

Buelow, J. M., Hinkle, J. L., \& McNett, M. (2016). Reliability and Validity for Neuroscience Nurses. Journal of Neuroscience Nursing, 48(5):238-241. https://doi.org/10.1097/JNN.0000000000000239.

Hill, M., Roshon, K., Bladen, C., Haley, E., Jr., McClelland, J., \& Suter, M. (2020). Decreasing Door-to-Groin Puncture Times in a Nonacademic Comprehensive Stroke Center. Journal of Neuroscience Nursing, 52(3):132-135. https://doi.org/10.1097/jnn.0000000000000505

Krishnamurthi, R. V., Feigin, V. L., Forouzanfar M. H., Mensah, G., Connor, M., Bennett, D., Moran, A., Sacco, R., Anderson, L., Truelsen, T., O'Donnell, M., Venketasubramanian, N., Barker-Collo, S., Lawes, C., Wang, W., Shinohara, Y., Witt, E., Ezzati, M., Naghavi, M., Murray, C. (2013). Global and regional burden of first-ever ischaemic and haemorrhagic stroke during 1990-2010: findings from the Global Burden of Disease Study 2010. The Lancet Global Health, 1(5):e259-281. https://doi.org/10.1016/S2214-109X(13)70089-5

Lakomkin, N., Dhamoon, M., Carroll, K., Singh, I., Tuhrim, S., Lee, J., Fifi, J., Mocco, J. (2019). Prevalence of large vessel occlusion in patients presenting with acute ischemic stroke: a 10-year systematic review of the literature. Journal of Neurointerventional Surgery, 11(3):241-245. https://doi.org/10.1136/neurintsurg-2018-014239

Lawrence, E., Merbach, D., Thorpe, S., Llinas, R. H., \& Marsh, E.B. (2018). Streamlining the Process for Intravenous Tissue Plasminogen Activator. Journal of Neuroscience Nursing, 50(1):37-41. https://doi.org/10.1097/JNN.0000000000000337

Middleton, S., Grimley, R., \& Alexandrov, A. W. (2015). Triage, Treatment, and Transfer. Stroke, 46(2):e18e25. https://doi.org/10.1161/STROKEAHA.114.006139

Powers, W. J., Rabinstein, A. A., Ackerson, T., Adeoye, O., Bambakidis, N., Becker, K., Biller, J., Brown, M., Demaerschalk, B., Hoh, B., Jauch, E., Kidwell, C., Leslie-Mazwi, T., Ovbiagele, B., Scott, P., Sheth, K., Southerland, A., Summers, D., Tirschwell, D. (2019). Guidelines for the Early Management of Patients With Acute Ischemic Stroke: 2019 Update to the 2018 Guidelines for the Early Management of Acute Ischemic Stroke: A Guideline for Healthcare Professionals From the American Heart Association/American Stroke Association. Stroke, 50(12):e344-e418. https://doi.org/10.1161/STR.0000000000000211

Rennert, R. C., Wali, A. R., Steinberg, J. A., Santiago-Dieppa, D., Olson, S., Pannell, J. S., \& Khalessi, A. (2019). Epidemiology, Natural History, and Clinical Presentation of Large Vessel Ischemic Stroke. Neurosurgery, 85(suppl_1):S4-S8. https://doi.org/10.1093/neuros/nyz042

Smith, E. E., Kent, D. M., Bulsara, K. R., Leung, L., Lichtman, J., Reeves, M., Towfighi, A., Whiteley, W., \& Zahuranec, D. (2018). Accuracy of Prediction Instruments for Diagnosing Large Vessel Occlusion in Individuals With Suspected Stroke: A Systematic Review for the 2018 Guidelines for the Early Management of Patients With Acute Ischemic Stroke. Stroke, 49(3):e111-e122. https://doi.org/10.1161/STR.0000000000000160

Smith, E., Shobha, N., Dai, D., Olson, D., Reeves, M., Saver, J., Fernandez, A., Peterson, E., Fonarow, G., \& Schwamm, L. (2013). A risk score for in-hospital death in patients admitted with ischemic or hemorrhagic stroke. Journal of the American Heart Association, 2(1), e005207. https://doi.org/10.1161/JAHA.112.005207.

Smith, W. S., Lev, M. H., English, J. D., Camargo, E., Chou, M., Johnston, S., Gonzales, G., Schaefer, P., Dillon, W., Koroshtetz, W., Furie, K. (2009). Significance of Large Vessel Intracranial Occlusion 
Causing Acute Ischemic Stroke and TIA. Stroke, 40(12):3834-3840. https://doi.org/10.1161/STROKEAHA.109.561787

Tavakol, M., \& Dennick, R. (2011). Making sense of Cronbach's alpha. International Journal of Medical Education, 2:53-55. https://doi.org/10.5116/ijme.4dfb.8dfd

Teleb, M. S., Ver Hage, A., Carter, J., Jayaraman, M. V., \& McTaggart, R.A. (2017). Stroke vision, aphasia, neglect (VAN) assessment-a novel emergent large vessel occlusion screening tool: pilot study and comparison with current clinical severity indices. Journal of Neurointerventional Surgery, 9(2):122126. https://doi.org/10.1136/neurintsurg-2015-012131

Ver Hage, A., Teleb, M., \& Smith, E. (2018).An Emergent Large Vessel Occlusion Screening Protocol for Acute Stroke: A Quality Improvement Initiative. Journal of Neuroscience Nursing, 50(2):68-73. https://doi.org/10.1097/JNN.0000000000000346

Zhu, W., Churilov, L., Campbell, B.C., LIn, M., Liu, X., Davis, S., Yan, B. (2014). Does large vessel occlusion affect clinical outcome in stroke with mild neurologic deficits after intravenous thrombolysis? Journal of Stroke and Cerebrovascular Disease, 23(10):2888-2893. https://doi.org/10.1016/j.jstrokecerebro vasdis.2014.07.018

\section{About the Authors}

Daniel Nyancho, MPH, is a Research Intern with the Neuroscience Nursing Research Center at the University of Texas Southwestern (UTSW) in Dallas, TX USA.

Ryan Cheung, DO, is a Vascular Neurology Fellow at UTSW

Aardhra M. Venkatachalam, MPH, is a Research Data Specialist at UTSW

Folefac D. Atem, PhD, is an Associate Professor of Biostatistics at the University of Texas Health Science Center in Houston. TX USA

Sonja E. Stutzman, PhD, is a Research Program Manager at UTSW

DaiWai M. Olson, PhD, RN, FNCS, is a Professor of Neurology at UTSW

Deborah Diercks, MD, is a Professor, and the Chair of Emergency Medicine at UTSW.

Mark Johnson, MD, is a Professor of Neurology at UTSW

Roberta Novakovic-White, MD, is an Associate Professor of Neuroradiology at UTSW 